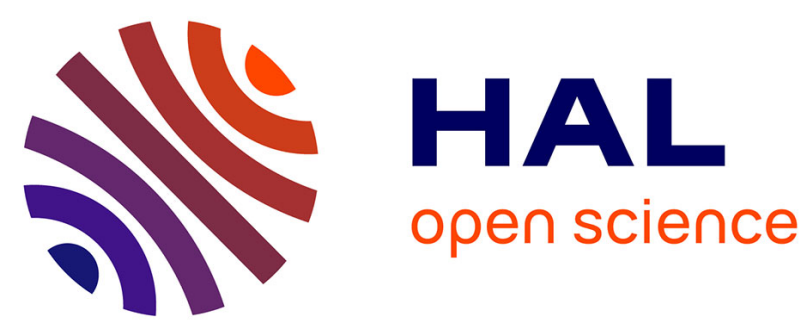

\title{
Stable vortex solitons in the Ginzburg-Landau model of a two-dimensional lasing medium with a transverse grating
}

Hervé Leblond, Boris Malomed, Dumitru Mihalache

\section{- To cite this version:}

Hervé Leblond, Boris Malomed, Dumitru Mihalache. Stable vortex solitons in the Ginzburg-Landau model of a two-dimensional lasing medium with a transverse grating. Physical Review A : Atomic, molecular, and optical physics [1990-2015], 2009, 80 (3), pp.033835. 10.1103/PhysRevA.80.033835 . hal-03423791

\section{HAL Id: hal-03423791 \\ https://univ-angers.hal.science/hal-03423791}

Submitted on 10 Nov 2021

HAL is a multi-disciplinary open access archive for the deposit and dissemination of scientific research documents, whether they are published or not. The documents may come from teaching and research institutions in France or abroad, or from public or private research centers.
L'archive ouverte pluridisciplinaire HAL, est destinée au dépôt et à la diffusion de documents scientifiques de niveau recherche, publiés ou non, émanant des établissements d'enseignement et de recherche français ou étrangers, des laboratoires publics ou privés. 


\title{
Stable vortex solitons in the Ginzburg-Landau model of a two-dimensional lasing medium with a transverse grating
}

\author{
Hervé Leblond, ${ }^{1}$ Boris A. Malomed, ${ }^{2}$ and Dumitru Mihalache ${ }^{3}$ \\ ${ }^{1}$ Laboratoire POMA, FRE 2988, Université d'Angers, 2 Boulevard Lavoisier, 49000 Angers, France \\ ${ }^{2}$ Department of Physical Electronics, Faculty of Engineering, Tel Aviv University, Tel Aviv 69978, Israel \\ ${ }^{3}$ Horia Hulubei National Institute for Physics and Nuclear Engineering (IFIN-HH), 407 Atomistilor, \\ Magurele-Bucharest 077125, Romania \\ (Received 17 July 2009; published 25 September 2009)
}

\begin{abstract}
We introduce a two-dimensional model of a laser cavity based on the complex Ginzburg-Landau equation with the cubic-quintic nonlinearity and a lattice potential accounting for the transverse grating. A remarkable fact is that localized vortices, built as sets of four peaks pinned to the periodic potential, may be stable without the unphysical diffusion term, which was necessary for the stabilization in previously studied models. The vortices are chiefly considered in the onsite (rhombic) form, but the stabilization of offsite vortices (squareshaped ones) and quadrupoles is demonstrated too. Stability regions for the rhombic vortices and fundamental solitons are identified in the model's parameter space, and scenarios of the evolution of unstable vortices are described. An essential result is a minimum strength of the lattice potential which is necessary to stabilize the vortices. The stability border is also identified in the case of the self-focusing quintic term in the underlying model, which suggests a possibility of the supercritical collapse. Beyond this border, the stationary vortex turns into a vortical breather, which is subsequently replaced by a dipolar breather and eventually by a single-peak breather.
\end{abstract}

DOI: 10.1103/PhysRevA.80.033835

PACS number(s): 42.65.Tg, 42.65.Sf, 47.20.Ky

\section{INTRODUCTION AND THE MODEL}

A generic model of the pattern formation in onedimensional (1D) and multidimensional lasing media is based on the complex Ginzburg-Landau (CGL) equation with the cubic-quintic (CQ) combination of nonlinear gain and loss terms, which are added to the linear loss [1]. The CQ nonlinearity makes it possible to create solitary pulses, alias dissipative solitons [2], sitting on the stable zero background. In fact, CGL equations, including those of the CQ type, find applications in many areas beyond the framework of optics, and they have also drawn a great deal of interest as general models of nonlinear dissipative media [3].

A challenging issue is the search for stable dissipativesoliton solutions of two- and three-dimensional (2D and 3D) versions of CGL equations. In that case, the potential instabilities inherited from the 1D case, such as the failure of the cubic gain (in the case of the CQ equation) to balance the combined linear and quintic loss terms, are exacerbated by the possibility of the collapse driven by the cubic selffocusing term. As concern solitons with the embedded vorticity (shaped as vortex rings), they are additionally vulnerable to azimuthal perturbations that tend to split them $[4,5]$. In fact, the CGL equation of the CQ type was originally introduced by Petviashvili and Sergeev [6], with the purpose to develop a model admitting stable localized patterns. Then, solutions for solitary pulses had been analyzed in detail in the $1 \mathrm{D}$ version of this equation [7]. Further, stable 2D vortical dissipative solitons (alias spiral solitons, so called because of the shape of the constant-phase lines in them), with vorticity (topological charge) $S=1$ and 2, were found in Ref. [8]. Stable 3D (spatiotemporal) fundamental solitons, with $S=0$ [9-11], and two-soliton complexes, including rotating ones [12], have been reported in models of lasing media based on the CQ CGL equation in the full 3D form. Recent works were dealing with 3D dissipative vortical solitons [13] and collisions between them [14].

The general form of the two-dimensional CQ CGL equation for the amplitude of the electromagnetic field, $u(x, y, z)$, which evolves along transmission distance $z$ in a bulk lasing medium with transverse coordinates $(x, y)$, is [13]

$$
\frac{\partial u}{\partial z}=\left[-\delta+\left(\frac{i}{2}+\beta\right) \nabla_{\perp}^{2}+(i+\varepsilon)|u|^{2}-(i \nu+\mu)|u|^{4}\right] u,
$$

where $\nabla_{\perp}^{2}$ is the Laplacian acting on $x$ and $y$, the coefficients which are scaled to be $1 / 2$ and 1 account for the diffraction in the transverse plane and the self-focusing Kerr nonlinearity, positive constants $\delta, \varepsilon$, and $\mu$ represent the linear loss, cubic gain, and quintic loss, respectively, and the self-defocusing or self-focusing quintic correction to the Kerr nonlinearity is represented by $\nu>0$ or $\nu<0$. Note that, in the conservative counterpart of the CQ CGL equation, i.e., the nonlinear Schrödinger (NLS) equation with the CQ nonlinearity, the quintic term must be self-defocusing (in the $2 \mathrm{D}$ and $3 \mathrm{D}$ settings) to arrest the collapse driven by the self-focusing cubic nonlinearity $[4,15]$. However, it was shown in Ref. [13] that multidimensional fundamental and vortical dissipative solitons may remain stable with the self-focusing sign of the quintic term, the collapse being precluded by the action of the quintic term in the dissipative part of the equation.

Actually, the physical meaning of all terms in Eq. (1) is clear except for the one coming with coefficient $\beta$, which represents an effective spatial diffusivity. This term is known in some specific models of large-aspect-ratio laser cavities, just above the first lasing threshold. Those models are based on the complex Swift-Hohenberg equation [16], which re- 
duces to the CGL equation if the fourth-order spatial derivatives are neglected.

In the general case, the diffusivity term is artificial as an ingredient of the two-dimensional CGL model of the laser cavity. Nevertheless, it was concluded in Ref. [13] that $\beta$ $>0$ was a necessary condition for the stability of vortex dissipative solitons in the multidimensional setting, while fundamental solitons might be stable at $\beta=0$. Thus, an important issue is to find a physically relevant modification of the 2D and 3D CGL models, with $\beta=0$ (without the unphysical diffusivity), which would support stable localized vortices. The objective of the present work is to demonstrate that this purpose can be achieved by adding a transverse periodic potential to Eq. (1), i.e., replacing that equation by

$$
\frac{\partial u}{\partial z}=\left[-\delta+i V(x, y)+\frac{i}{2} \nabla_{\perp}^{2}+(i+\varepsilon)|u|^{2}-(i \nu+\mu)|u|^{4}\right] u,
$$

with a potential that can be induced by a grating, i.e., periodic local modulation of the refractive index in plane $(x, y)$. In the simplest case, the effective potential of the grating may be approximated by

$$
V(x, y)=V_{0}[\cos (2 x)+\cos (2 y)],
$$

where the scaling invariance of Eq. (2) was employed to fix the transverse periods of the potential in the $x$ and $y$ directions to be $\pi$, assuming that these periods are equal.

In bulk photonic media, permanent gratings can be written by means of a known optical technology [17]. In addition, in photorefractive crystals virtual photonic lattices may be temporarily induced by pairs of extra laser beams illuminating the sample in the directions of $x$ and $y$ in the ordinary polarization, while the probe beam is launched along axis $z$ in the extraordinary polarization [18].

It is also relevant to notice that CGL-type equations describe laser cavities, where the mode-locked optical signal performs periodic circulations, as averaged models (see, e.g., the derivation for ring fiber lasers [19]). Therefore, in the general case, the transverse grating is not required to fill the entire cavity. A localized component, such as a holographic phase plate, might be sufficient, provided that it induces the same phase distortion as potential $V(x, y)$ in one circulation.

Stationary solutions to Eq. (2) are sought for as

$$
u(x, y, z)=e^{i k z} U(x, y),
$$

with real propagation constant $k$ and complex function $U(x, y)$ satisfying equation

$$
i k U=\left[-\delta+i V(x, y)+\frac{i}{2} \nabla_{\perp}^{2}+(i+\varepsilon)|U|^{2}-(i \nu+\mu)|U|^{4}\right] U .
$$

In the presence of the periodic (lattice) potential, stable vortices are built as compound objects, consisting of four separate peaks of the local power (density). The four peaks are set in four cells of the lattice (in fact, each peak may be approximately considered as a tightly localized fundamental soliton). Two basic configurations of the so constructed vor- tices are known: "rhombuses," alias onsite vortices, with a nearly empty cell surrounded by the filled ones [20,21], and "squares," alias offsite vortices, which feature a densely packed set of four filled cells, without an empty site in the middle [22]. The topological charge (vorticity) of these patterns is provided by phase shift $\pi / 2$ between adjacent peaks, which corresponds to the total phase circulation of $2 \pi$ around the pattern, as it should be in vortices with topological charge $S=1$.

The stabilization of vortex solitons by periodic potentials was predicted in various settings [20-24]. In the experiment, stable localized vortices with $S=1$ were created in the abovementioned photorefractive crystals equipped with the virtual photonic lattice [25]. In a certain sense, the lattice potential gives rise to an effective nonlocality in the medium. In this connection, it is relevant to mention that the stabilization of vortex solitons by nonlocal nonlinearities in uniform media was also predicted in a number of theoretical works [26] and demonstrated in an experiment [27].

In this work, we chiefly focus on the identification of the stability region for rhombic vortices in the framework of model (2), which are usually most stable objects [21] although stable squares will be presented too. In addition to the compound vortices, four peaks arranged in the form of a rhombus or square may represent a quadrupole, with alternating signs of the peaks [21]. We will produce examples of stable rhombic quadrupoles in the present model.

Besides the stability of the compound vortices supported by the lattice, another central topic of this work is the extension of the stability to the above-mentioned case of $\nu<0$, i.e., the self-focusing quintic nonlinearity, which is known to cause the supercritical collapse in 2D conservative media [28]. It will be demonstrated that, if other parameters are fixed, there is a certain negative critical value of $\nu$ up to which the vortices remain stable. Beyond the critical point, the stationary vortices are replaced by oscillating vortical breathers, which perform periodic oscillations, keeping the phase structure corresponding to the vortices. At still larger values of $(-\nu)$, the vortex breather becomes unstable, being replaced by a dipolar breather, which is built of two peaks filling cells which sit on the diagonal of the lattice. Further, at much larger $-\nu$, the dipole gets destabilized too, being replaced by a single-peak breather and eventually all stable patterns disappear.

The stability of various patterns considered in this paper is identified by way of systematic direct simulations of their self-trapping from localized inputs and subsequent tests of the evolution under the action of added random perturbations. In dissipative models, this approach is sufficient to unambiguously determine the stability of various patterns, while in their conservative counterparts an accurate computation of stability eigenvalues for modes of small perturbations is necessary, as perturbed stable solutions keep oscillating indefinitely long rather than relaxing back to the stationary shape (see, e.g., Refs. [20-24], [26], and [28-31]).

The rest of the paper is organized as follows. Before addressing the main topic of the stability of vortices, in Sec. II we consider fundamental (single-peak) 2D dissipative solitons in the present model. The stationary vortex solitons (chiefly rhombuses) are considered in Sec. III, where we also 
present examples of stable square-shaped vortices and quadrupoles. Vortical and dipolar breathers are reported in Sec. IV, and Sec. V concludes the paper.

\section{FUNDAMENTAL DISSIPATIVE SOLITONS}

\section{A. Mode of simulations}

The existence of stable fundamental solitons in the present model is not surprising, as they are known in the 2D CGL equation corresponding to Eq. (2) without the lattice potential, $V=0$ [8] (unlike localized vortices, which cannot be stable in that case). Nevertheless, 2D fundamental solitons in the CGL equation with the lattice potential were not studied before, and it is relevant to report basic results for them here-in particular, for the sake of comparison with localized vortices, which will be considered below.

All localized patterns-fundamental solitons, vortices, quadrupoles, and dipoles-studied in this work were found as established objects (attractors of the dissipative model) by means of direct simulations of Eq. (2) based on the use of the fourth-order Runge-Kutta algorithm. Laplacian $\Delta u$ was computed using a centered five-point finite-difference formula. The sizes of the integration domain were $140 \times 140$ for the fundamental solitons and $200 \times 200$ for vortices, including an absorption layer ten points wide adjacent to each edge of the domain, which was introduced to prevent the reflection of radiation waves shed off by evolving localized objects. After a particular stationary solution was found, it was used as the initial configuration for a new run of simulations, with slightly modified parameters of Eq. (2), with the aim to find an attractor corresponding to the modified parameters. We note that, in the case of a sufficiently strong self-focusing quintic nonlinearity, i.e., relatively large $(-\nu)$ in Eq. (2) (see Sec. IV below), the "established object," to which the direct simulations converged, was not a stationary pattern but rather a robust well-localized breather.

In the case when the simulations converged to stationary established states, their stability was additionally tested by adding to them random perturbations at the amplitude level of $10 \%$ and running the subsequent simulations-typically up to $z=400$ for fundamental solitons and $z=1400$ for vortices. The evolution of the amplitude of the pattern [the largest value of $|U(x, y)|]$, instantaneous propagation number $k(z)$ [extracted from the numerical data with the help of Eq. (5) and averaging over space], and the total power,

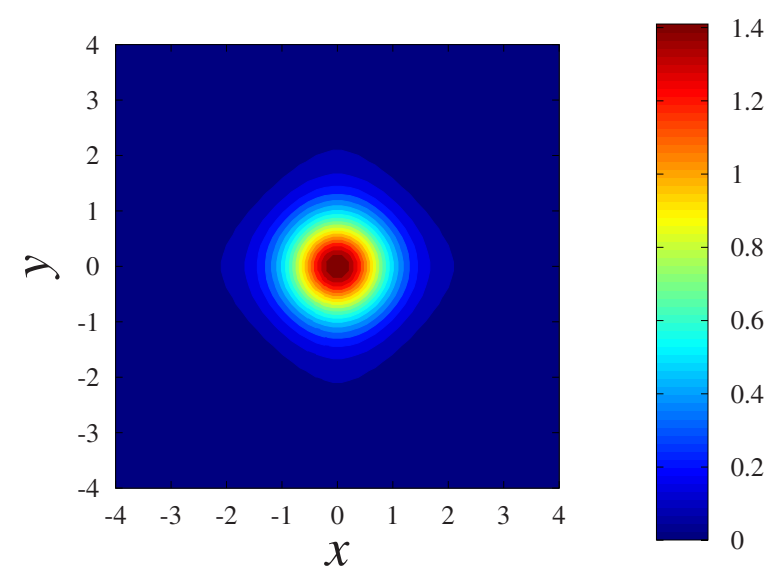

FIG. 1. (Color online) The stable fundamental soliton at parameter values chosen as per Eq. (7).

$$
P=\iint|u(x, y, z)|^{2} d x d y,
$$

were monitored in the course of the simulations. The solution was identified as stable if the amplitude and shape of the pattern relaxed back to the unperturbed configuration.

It is necessary to stress that, along with completely stable stationary solutions, weakly unstable ones were found too. Indeed, after the stationary solution has been perturbed by the addition of white noise, it quickly relaxes to the unperturbed state. Then the instability eventually develops, however in some cases it is extremely slow: the energy may have grown for less than $10 \%$ after propagation over $z=1000$. Such states are attractors in the sense that the input beams originally converge to them. The "lifetime" of the unstable states diverges at boundaries of the stability domain, therefore there was a small uncertainty in locating the stability boundaries using results of the direct simulations.

In terms of the physical applications, unstable localized modes, as they are defined above, are meaningful objects in models of amplifying media, where the transmission distance is always finite; hence the existence length of the unstable soliton may be readily made larger than the size of the medium. On the other hand, only truly stable solitons are relevant to models of mode-locked laser cavities, where the circular transmission of the light beam makes the effective propagation length infinitely large.
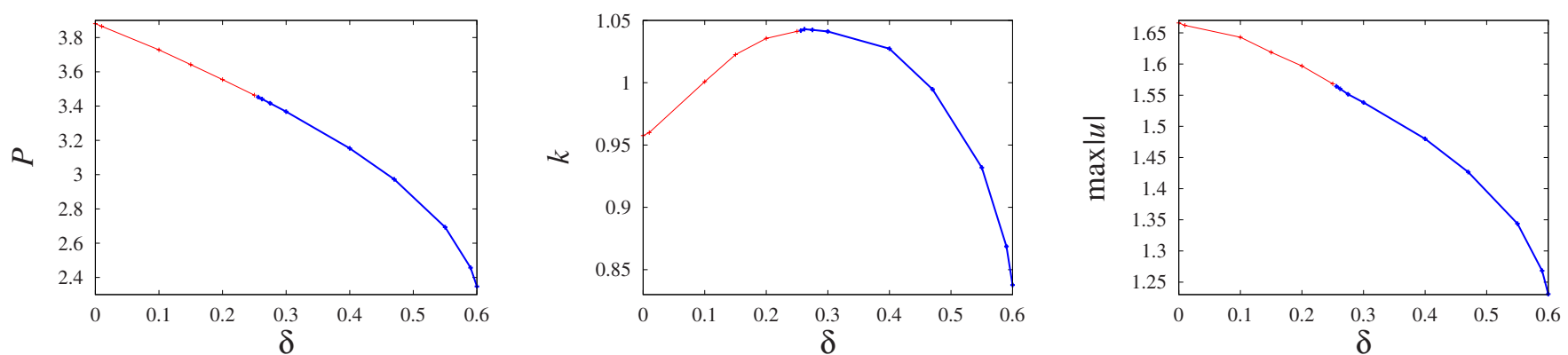

FIG. 2. (Color online) Characteristics of the fundamental soliton versus $\delta$. Red (thin) and blue (thick) segments depict unstable and stable soliton subfamilies. Crosses are data points representing numerical results. 

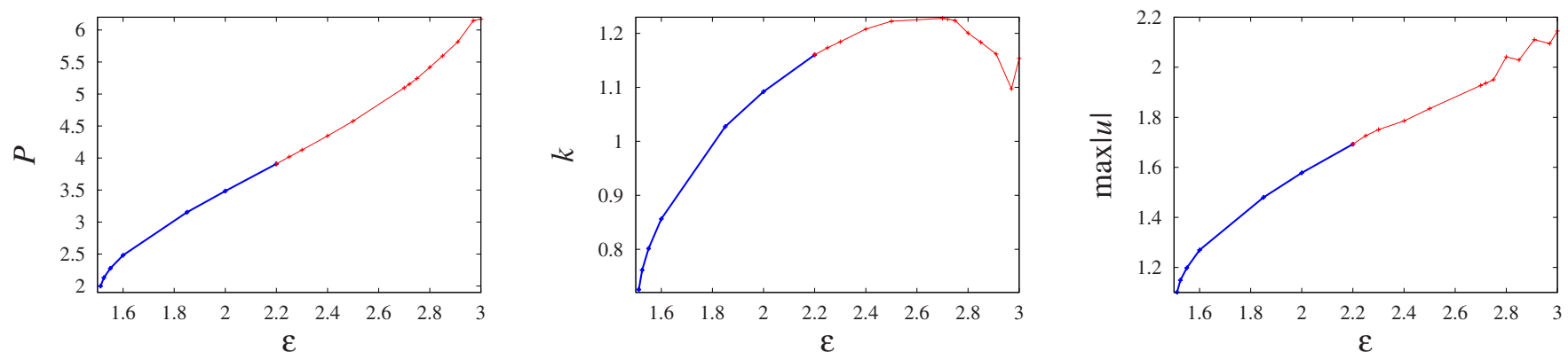

FIG. 3. (Color online) The same as in Fig. 2 but versus $\varepsilon$.

In those cases when localized modes could not self-trap in the course of the evolution or existed temporarily but eventually turned out to be unstable, the eventual state of the system might be either simply zero or a disordered pattern of speckles filling the entire integration domain (a typical example of the pattern can be seen below in the last panel of Fig. 13). Naturally, the decay to zero was observed when the dissipation was too strong versus the gain, i.e., coefficients $\delta$ and/or $\mu$ in Eq. (2) were too large or coefficient $\varepsilon$ was too small. In the opposite case, when the loss was too weak in comparison with the gain, the system would switch into the speckle pattern that filled out all lattice cells.

The simulations started from a stationary state found for parameter values

$$
\delta=0.4, \quad \varepsilon=1.85, \quad \mu=1, \quad \nu=0.1, \quad V_{0}=1
$$

in Eq. (2), which is a typical case that gives rise to stable solitons and vortices. Then, the analysis was extended by varying all the parameters until hitting a border at which the simulations would cease to converge to a localized state.

\section{B. Results for fundamental solitons}

Starting from an input in the form of a beam (in terms of laser cavities) with an isotropic Gaussian profile, it was straightforward to find a stable fundamental soliton. The stable fundamental soliton existing at parameter values given in Eq. (7) is shown on Fig. 1. Extending the analysis by varying parameters $\delta, \varepsilon, \mu, \nu$, and $V_{0}$, we have produced characteristics of the family of the fundamental solitons which show, in Figs. 2-6, their integral power [see Eq. (6)], propagation constant, and amplitude as functions of these parameters (in Figs. 5 and 6, the dependence of the ampli- tude on $\nu$ and $V_{0}$ is not shown, as the amplitude remains practically constant within the ranges of the variation in the parameters in those cases).

The figures explicitly display subfamilies of stable and unstable solitons (recall that unstable ones self-trap from the input beam but are eventually destroyed by perturbations). Further, except for the case of Fig. 6, neither stable nor unstable soliton exists outside of ranges of the parameters displayed in Figs. 2-5. Actually, at $\delta>\delta_{\max }=0.602 \pm 0.003$ (see Fig. 2), $\varepsilon<\varepsilon_{\min }=1.506 \pm 0.006 \quad$ (Fig. 3), $\mu>\mu_{\max }$ $=1.48 \pm 0.02$ (Fig. 4), and $\nu<\nu_{\min }=-0.605 \pm 0.005$ (Fig. 5), the system relaxes into the zero state (the critical values are given with error margins determined by the procedure of the identification of stable and unstable regions in the parameter space, as specified above). On the other hand, at $\varepsilon>\varepsilon_{\max }$ $=3.125 \pm 0.125, \quad \mu<\mu_{\min }=0.385 \pm 0.010, \quad$ and $\quad \nu>\nu_{\max }$ $=0.931 \pm 0.006$, the evolution leads to the establishment of a delocalized speckle pattern. A natural conclusion is that the subfamilies of stable and unstable solitons are bordered, respectively, by parameter regions where the system evolves to the zero state or to the speckle-filled one.

Intrinsic frontiers between the stable and unstable subfamilies are found at the following points: $\delta=0.253 \pm 0.003$ in Fig. $2, \varepsilon=2.22 \pm 0.03$ in Fig. 3, and $\mu=0.59 \pm 0.03$ in Fig. 4. The entire family shown in Fig. 5 is stable. As concerns the role of strength $V_{0}$ of the lattice potential, it does not impose any limitation on the stability of the fundamental solitons, i.e., they are stable not only in the interval displayed in Fig. 6 but actually at all values of $V_{0}$ that were considered. Note that stable 2D fundamental solitons in the uniform space, reported in previous works [8], correspond to point $V_{0}=0$ in Fig. 6.

For more negative $\nu$, namely $\nu_{\min }=-0.605 \pm 0.005>\nu$ $>\nu_{\min }^{\prime}=-0.94 \pm 0.015$, oscillating states of a single-peak
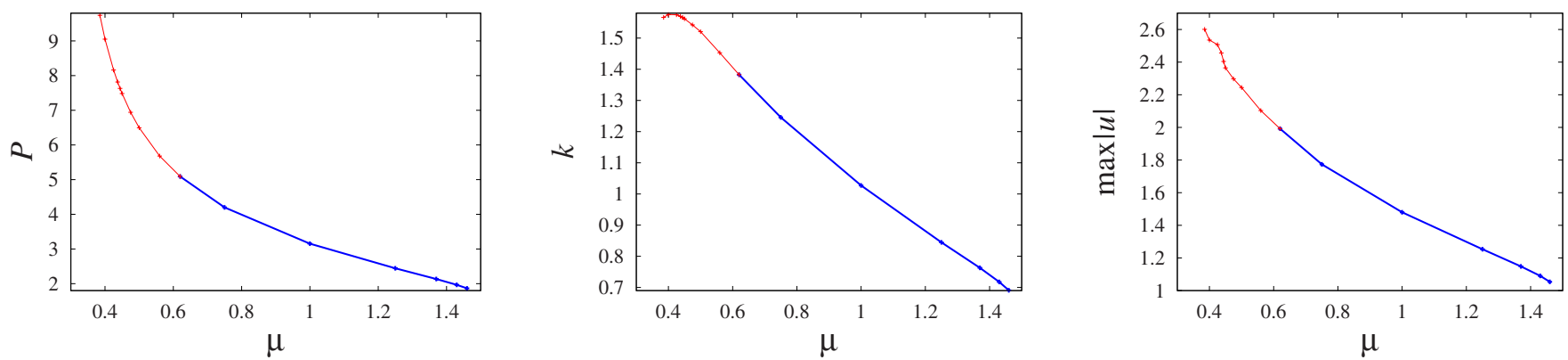

FIG. 4. (Color online) The same as in Fig. 2 but versus $\mu$. 


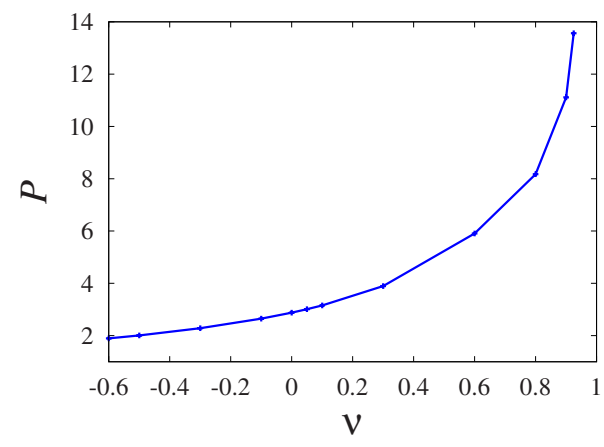

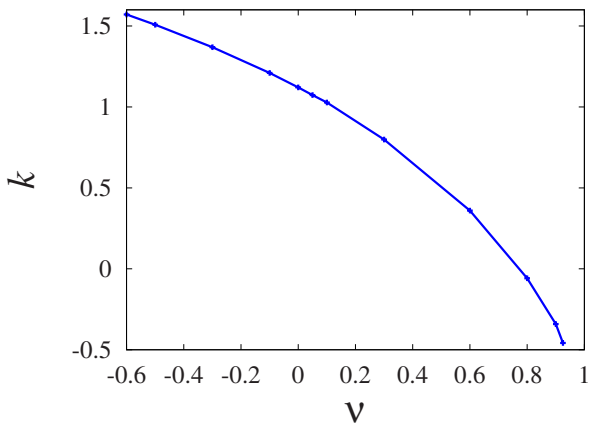

FIG. 5. (Color online) The two panels show the same as their left and middle counterparts as in Fig. 2 but versus $\nu$. The dependence of the amplitude on $\nu$ is not shown here, as this dependence is extremely weak. breather type are found. Such structures are studied below in the framework of vortex instabilities (see Sec. IV).

\section{VORTICES}

\section{A. Rhombus-shaped vortex solitons}

As mentioned in Sec. I, in this work we chiefly focus on the localized complexes with topological charge $S=1$ built of four tightly bound peaks that form a rhombus (onsite vortices). A set of images illustrating the distribution of the local amplitude in stationary rhombic vortices is presented in Fig. 7. In all cases, the phase shift between neighboring local maxima of the intensity is $\pi / 4$, matching the definition of the vortex with $S=1$. In the conservative system, due to the symmetry of the NLS equation, the amplitude pattern of the stable vortex is symmetric with respect to the symmetry axes of the potential $(x=0, y=0$, and $x= \pm y)$.

Careful examination of panels (c), (f), and (h) in Fig. 7 reveals that the vortex pattern features a weak spirality (alias "chirality" in the sense that the vortex with $S=-1$ would feature the opposite spirality). In fact, the presence of the spirality is readily explained by the lack of the invariance of the CGL equation with respect to the complex conjugation (unlike the conservative NLS equation, where the conjugation may be compensated by the reversal of sign of the evolution variable, $z$ ). In fact, the spirality is a well-pronounced feature of vortex solutions to the CGL equations in the free $2 \mathrm{D}$ space [8].

\section{B. Stability limits for the vortex solitons}

Results concerning the stability of the vortex solitons against the variation in parameters $\delta, \varepsilon, \mu$, and $\nu$ are presented in Figs. 8-11 in essentially the same way as was done above for the fundamental solitons (cf. Figs. 2-5). In particu- lar, the intrinsic borders between subfamilies of stable and unstable vortices are found at $\delta=0.305 \pm 0.005$ in Fig. $8, \varepsilon$ $=2.12 \pm 0.03$ in Fig. 9, and $\mu=0.78 \pm 0.03$ in Fig. 10 .

Like in the case of the fundamental solitons, in these figures the stable and unstable subfamilies abut on parameter regions where the system evolves into the zero state or develops the delocalized speckle pattern, respectively. The former outcome of the evolution of input beams is observed at $\delta>\delta_{\max }=0.599 \pm 0.004$ in Fig. 8 , at $\varepsilon<\varepsilon_{\min }$ $=1.518 \pm 0.007$ in Fig. 9, and at $\mu>\mu_{\max }=1.48 \pm 0.03$ in Fig. 10. The delocalized pattern appears at $\delta<\delta_{\text {min }}$ $=0.15 \pm 0.03$ in Fig. 8 , at $\varepsilon>\varepsilon_{\max }=2.75 \pm 0.05$ in Fig. 9, at $\mu<\mu_{\min }=0.47 \pm 0.01$ in Fig. 10 , and at $\nu>\nu_{\max }$ $=0.585 \pm 0.004$ in Fig. 11. Notice that no unstable stationary vortex has been found when $\nu$ was varied.

In some cases, vortex solitons may become unstable in a very narrow interval adjacent to the region where the evolution leads to the decay to zero (a feature that was not found for fundamental solitons). This small interval is found about $\delta=0.602$ in Fig. 8 , about $\varepsilon=1.51$ in Fig. 9, and about $\mu$ $=1.5$ in Fig. 10. The accurate computation of the width of these narrow unstable stripes would require prohibitively long simulations.

Note that the range of coefficient $\nu$, which accounts for the conservative part of the quintic nonlinearity, is extended in Fig. 11 into the region of the quintic self-focusing $(\nu$ $<0)$ up to point

$$
\nu_{\min }=-0.515 \pm 0.015
$$

at which a different destabilization mechanism comes into play, replacing the stationary vortex by its breather counterpart, which, however, keeps the vorticity, i.e., remains an intrinsically coherent complex even if being nonstationary (oscillating). This issue and the evolution of the vortical

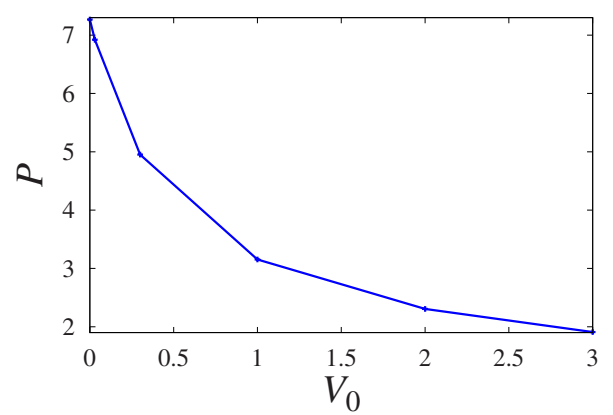

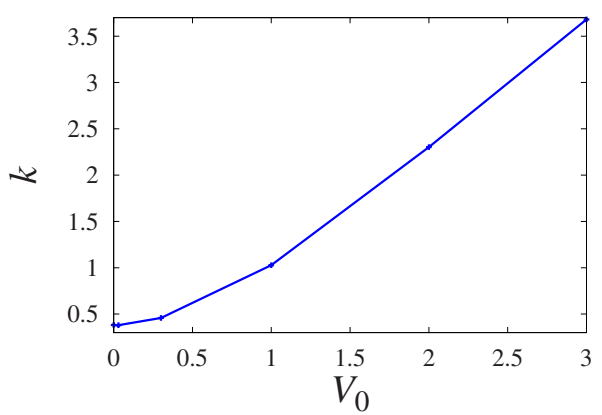

$033835-5$
FIG. 6. (Color online) The same as in Fig. 5 but versus $V_{0}$. 

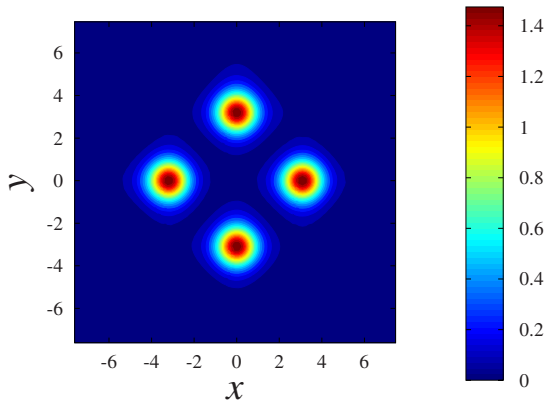

(a) $\delta=0.31$

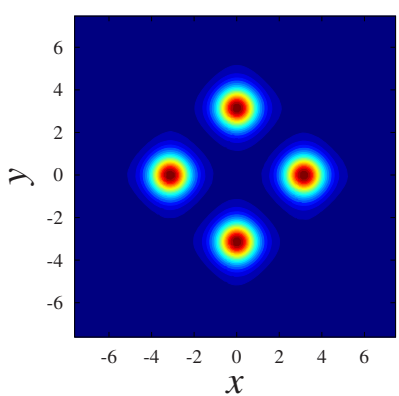

(d) $\varepsilon=2.1$

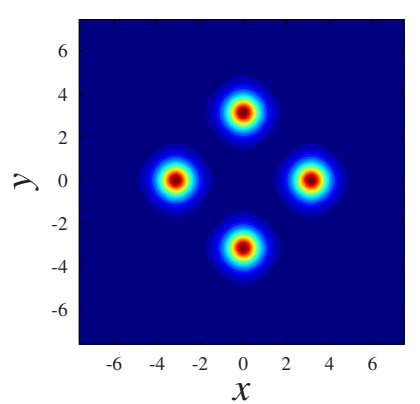

(g) $\nu=-0.5$

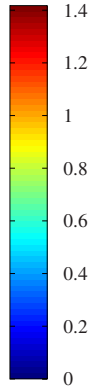

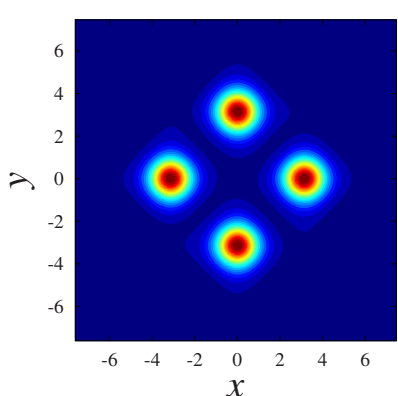

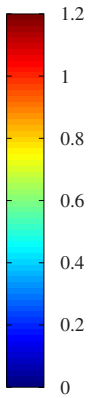

(b) $\delta=0.595$

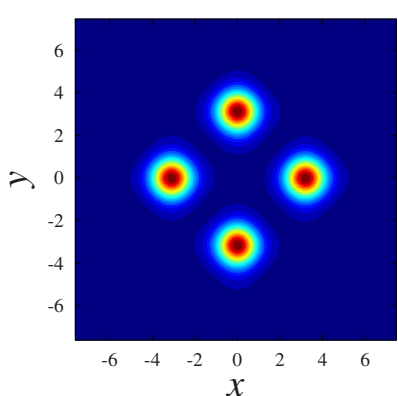

(e) $\mu=0.8$
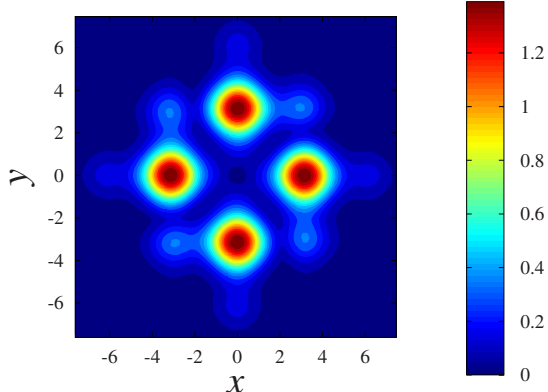

(h) $\nu=0.581$

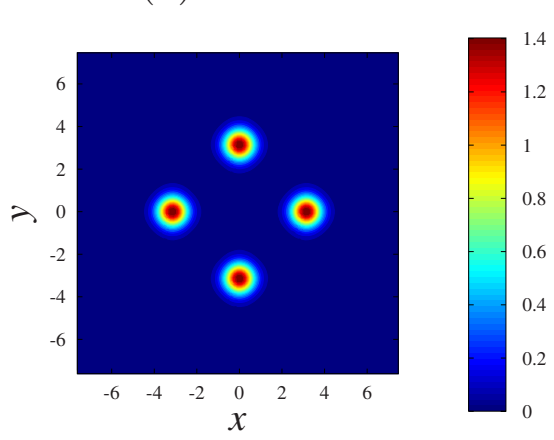

(j) $V_{0}=4$

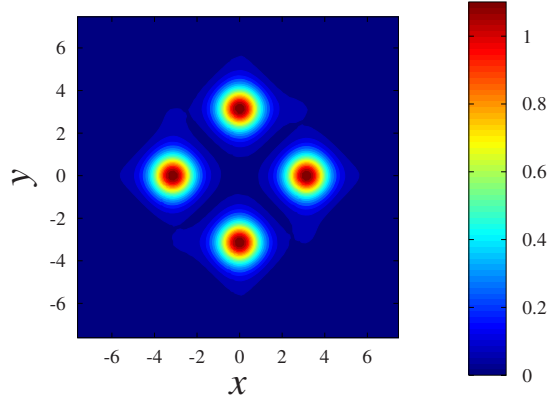

(c) $\varepsilon=1.525$

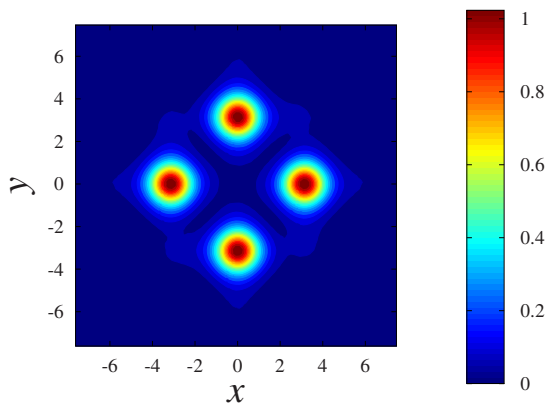

(f) $\mu=1.45$

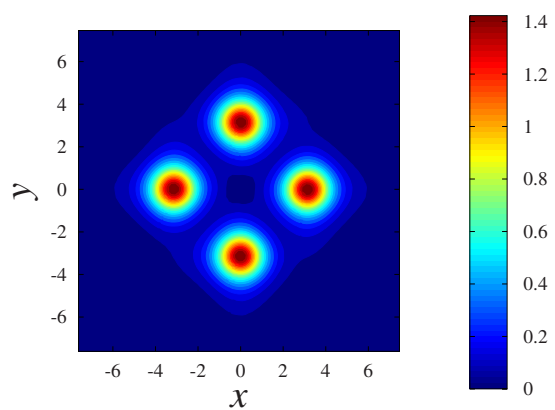

(i) $V_{0}=0.42$ 

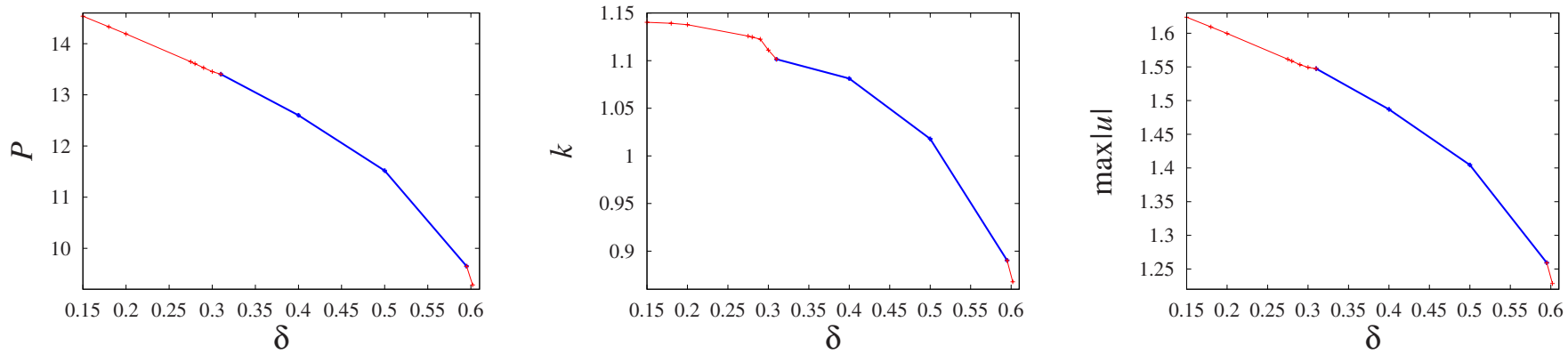

FIG. 8. (Color online) The same as in Fig. 2 but for the family of rhombic (onsite) vortices.

uniform space, vortex solitons can never be stable in the 2D (or 3D) CGL equation without the diffusivity term, which is usually unphysical in models of lasing media]. The most essential finding reported in Fig. 12 is that the stabilization takes place if the lattice is strong (deep) enough, viz., at $V_{0}$ $>\left(V_{0}\right)_{\min }=0.38 \pm 0.04$ (the stability of the vortex was verified by direct simulations up to $V_{0}=4$ and there is no reason to expect that the vortex will not be stable in a still deeper lattice). Finally, we note that, as well as the case of the fundamental solitons, Figs. 11 and 12 do not show the dependence of the amplitude on $\nu$ and $V_{0}$ because the amplitude remains almost constant within the parameter ranges shown in the figures.

\section{Instability modes}

Because the stabilization of the vortices is a central topic of this work, it is relevant to describe typical modes of the development of their instability in those cases when the vortices are unstable. In the 2D CGL equation in the uniform space (without the lattice potential), where the localized vortices can never be stable, the instability splits them into a few fundamental solitons [8] (this scenario, determined by the instability of the axially symmetric ring-shaped vortex against azimuthal perturbations, is roughly the same as in conservative models, which are based on equations of the NLS type [4,29]).

Systematic simulations of the present model reveal two essentially different scenarios of the evolution of unstable vortices. One of them is observed when the strength of the lattice potential, $V_{0}$, is large enough for the stabilization of the vortex, but other parameters are out of the respective stability range. A typical example of the development of this instability is displayed in Fig. 13 for $\varepsilon=2.4$, the other param- eters being as in Eq. (7) (as seen from Fig. 9, this value indeed falls into the instability interval).

The instability scenario presented in Fig. 13 can be summarized as follows. The four peaks of the local power, which form the vortex, start to oscillate, at first synchronously, but then the oscillations become mutually incoherent. At the next stage, the peaks stretch into extended spots, and, eventually, the spots extend over the entire lattice, creating the speckle pattern. A variety of essentially the same scenario (not shown here) is observed, for instance, at $\delta=0.2$, with other parameters fixed as per Eq. (7) (this point indeed corresponds to the instability of the vortex, as seen from Fig. 8). The difference is that two power peaks tend to coalesce before suffering the stretching and the instability development is essentially slower.

A very different instability mode is observed when the strength of the lattice potential is insufficient for the stabilization of vortices. A typical example of that is displayed for $V_{0}=0.15$ in Fig. 14. This scenario also starts with oscillations of each power peak. This first stage of the unstable evolution is very long. Then, two peaks leave their original positions and merge. The fused peak and two others start erratic motion, then the double peak again splits into two, and, eventually, four mutually incoherent peaks continue a slow random walk across the lattice. Thus, in this case the pattern stays in the form of a set of four well-localized peaks and the transition to the speckled pattern does not take place.

\section{Examples of the square-shaped vortex and quadrupole}

As mentioned in Sec. I, the lattice potential in conservative systems (described by NLS equations) supports, in addition to the onsite (rhombus-shaped) vortices, their offsite counterparts built as squares and quadrupoles. The present
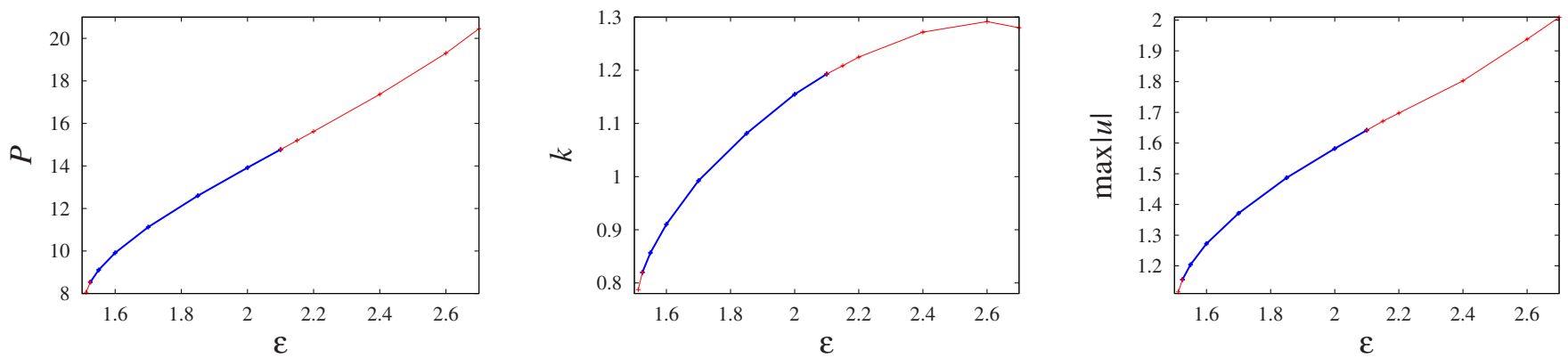

FIG. 9. (Color online) The same as in Fig. 3 but for the family of rhombic (onsite) vortices. 

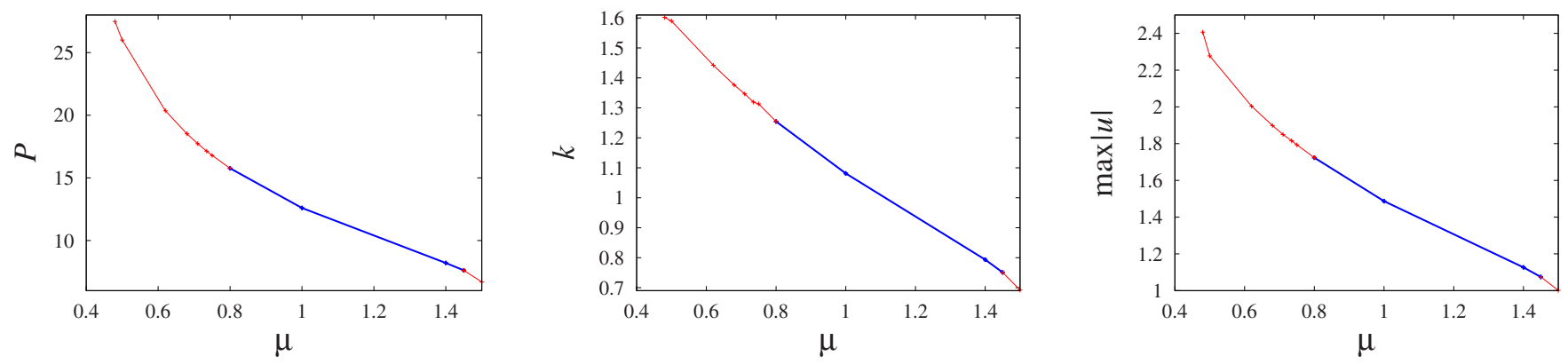

FIG. 10. (Color online) The same as in Fig. 4 but for the family of rhombic (onsite) vortices.

dissipative model also readily gives rise to stable patterns of these two types. To construct them, the fundamental soliton was approximated by a fitting formula,

$$
U(x, y)=\frac{2 A}{\cosh [a(x+y)]+\cosh [a(x-y)]},
$$

in which amplitude $A$ and inverse width $a$ are determined from numerical data. Then, quadrupoles and square-shaped vortices were constructed as the corresponding linear superpositions of position- and phase-shifted expressions taken as per Eq. (9). Finally, direct simulations produced established patterns of the types sought for (see typical examples in Figs. 15 and 16). The stability of these patterns against added random perturbations was also verified in direct simulations.

\section{VORTICES IN THE MODEL WITH THE SELF- FOCUSING QUINTIC NONLINEARITY}

It was shown in Fig. 11 that the family of rhombic vortices extends, as a stable one, into the region of the selffocusing conservative part of the quintic nonlinearity, up to a certain point [see Eq. (8)], if other parameters are fixed as in Eq. (7). This means that the quintic loss suppresses the trend to the supercritical collapse [28] despite the action of the quintic self-focusing term. Eventually, the increase in $-\nu$ leads to destabilization of the vortex at a point given by Eq. (8). Nevertheless, the vortex is not destroyed beyond this border. Instead, it turns into a vortical breather, with the heights of the four peaks featuring regular oscillations. As shown in Fig. 17, all the peaks oscillate quasiharmonically, with equal frequencies and amplitudes and phase shifts of $\pi / 2$ between the local oscillations, i.e., the same as phase shifts between the peaks which form a stable vortex. In turn, the phase shifts between values of the complex field at the peak points also start to oscillate, as one can conclude from Fig. 17, but the total phase circulation around the breather remains equal to $2 \pi$, i.e., the oscillations do not destroy the intrinsic coherence of the vortex (another example of a 2D system, although a discrete and conservative one, where the instability of stationary vortices does not destroy them but rather transforms these objects into vortical breathers, is the 2D Salerno model with the competition of onsite selffocusing and offsite self-defocusing cubic terms [30]). Additional simulations demonstrate that, in the region of its existence, the vortical breather is stable against random perturbations.

The vortical breather remains a robust and coherent dynamical complex approximately up to $\nu=-0.85$ if other parameters keep their values as fixed in Eq. (7). Then, this breather loses its stability, featuring a spontaneous transformation into a dipolar breather. The latter localized mode is composed of two harmonically oscillating peaks, which keep on average the phase shift between them close to $\pi$, although the instantaneous value of the phase difference oscillates, roughly, between $0.4 \pi$ and $1.2 \pi$ (see Fig. 18). As well as the

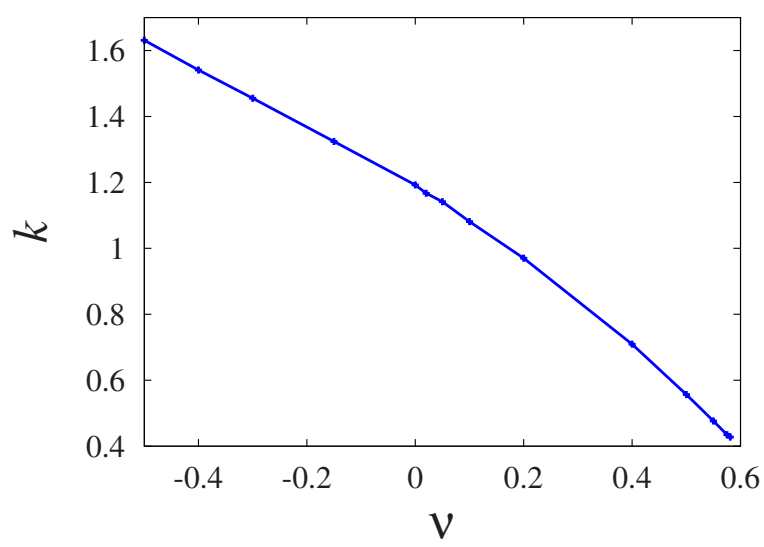

FIG. 11. (Color online) The same type of the dependences as in Fig. 5 but for the family of rhombic vortices. Note that values $\nu<0$, corresponding to the self-focusing quintic nonlinearity, are also included in this plot. It is extended up to a negative value of $\nu$ at which the stationary vortex loses its stability, being replaced by a vortical breather (see Sec. III C). 


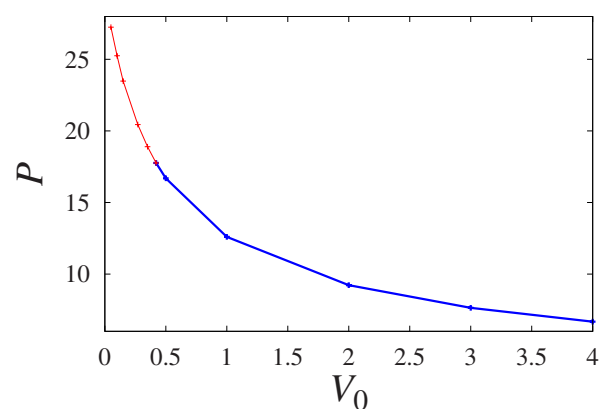

vortical breathers, the oscillating dipole maintains its integrity in the region where it exists, irrespective of the addition of random perturbations.

With the subsequent increase in $-\nu$, the oscillating dipole also loses its stability, being replaced by a single-peak breather (a pulsating counterpart of the fundamental soliton), whose shape and oscillations are displayed in Fig. 19. The

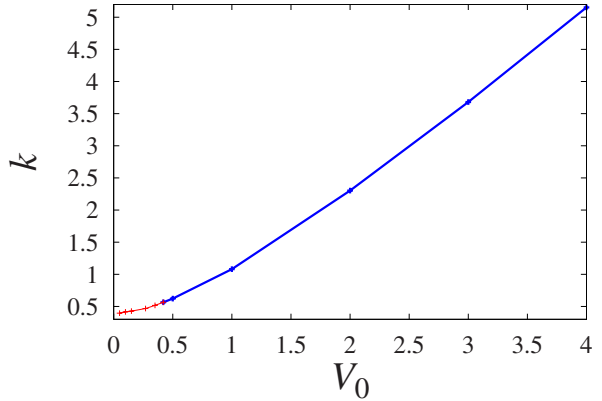

oscillations are strongly anharmonic but nevertheless regular and periodic.

Thus, we conclude that the increase in $-\nu$ gradually leads to the degradation of the shape of the breathing complexes from four peaks through two peaks to one. Nevertheless, a noteworthy fact is that four- and two-peak patterns, as long as they exist, maintain their intrinsic integrity, i.e., the vor-

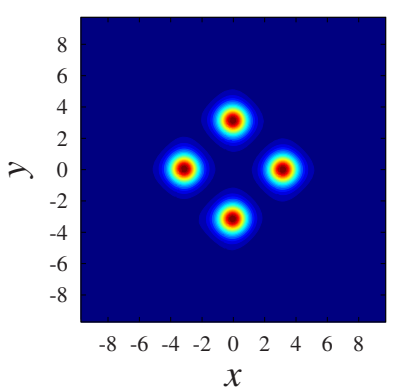

(a) $z=110.68$

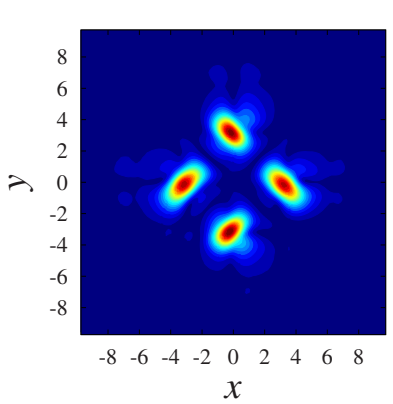

(d) $z=200.22$
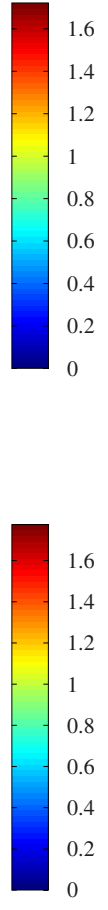

(b) $z=197.08$
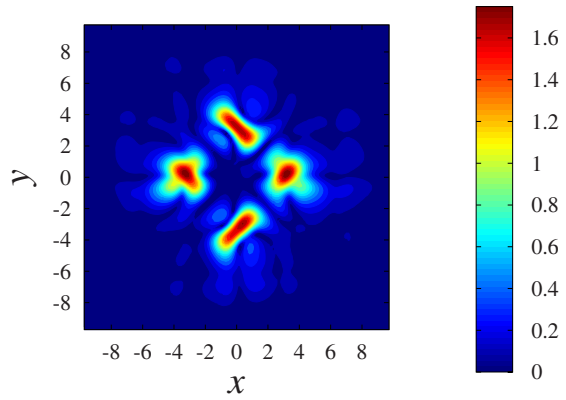

(e) $z=204.53$

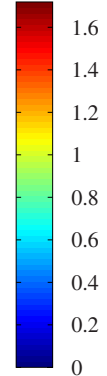

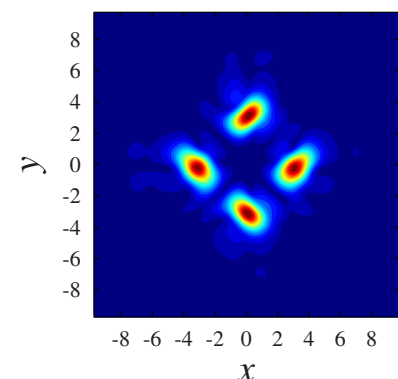

(c) $z=198.65$

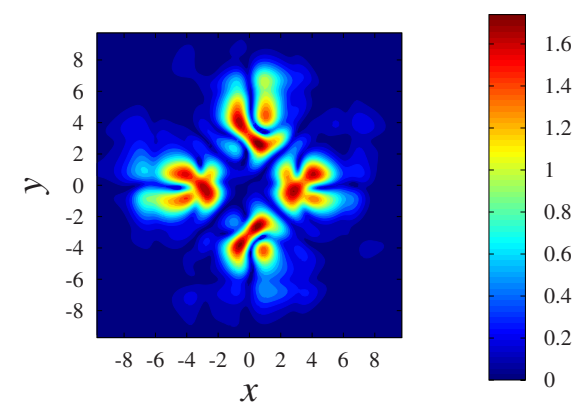

(f) $z=208.38$

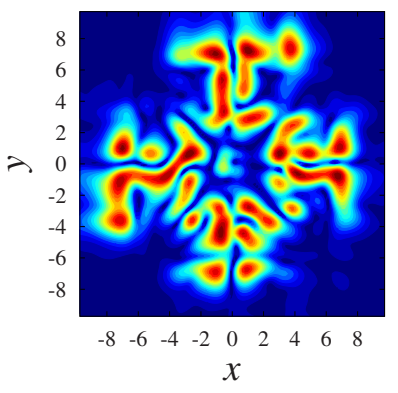

(g) $z=211.81$
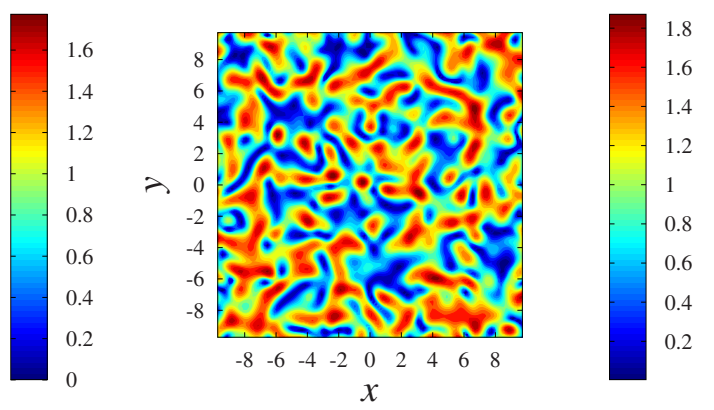

(h) $z=226.22$

FIG. 13. (Color online) Evolution of an unstable vortex with $\varepsilon=2.4$. Other parameters are chosen as in Eq. (7). 


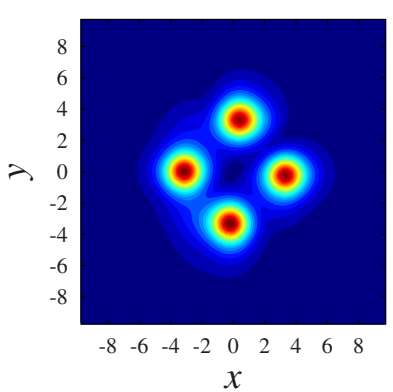

(a) $z=2567.3$

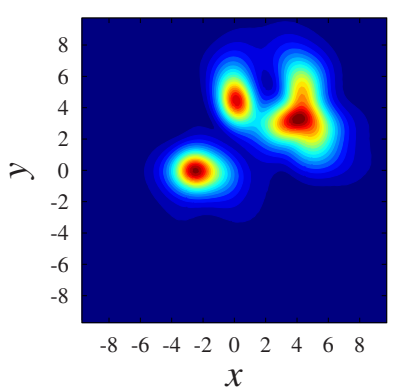

(d) $z=2605.1$

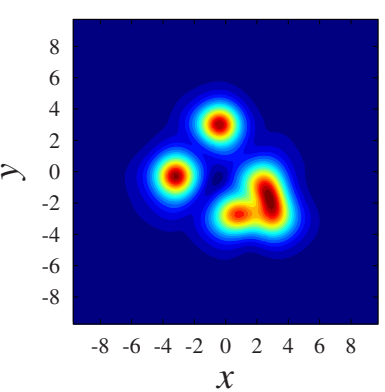

(b) $z=2576.7$
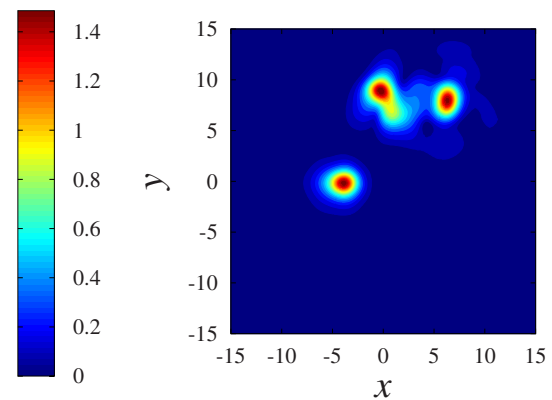

(e) $z=2624$

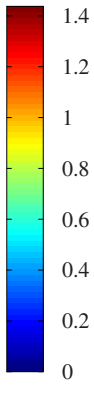

(g) $z=2671.1$
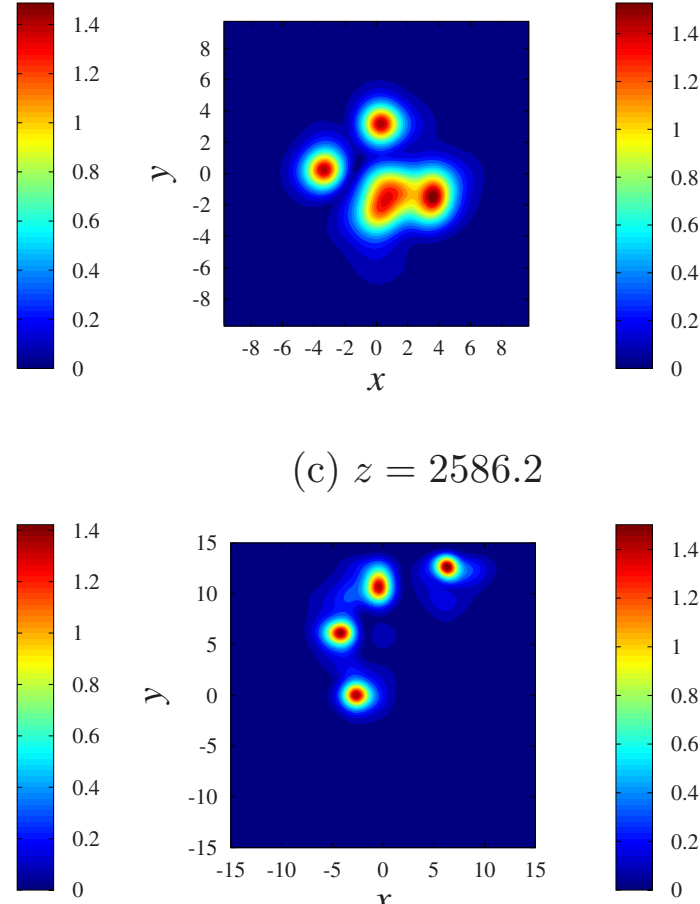

(c) $z=2586.2$

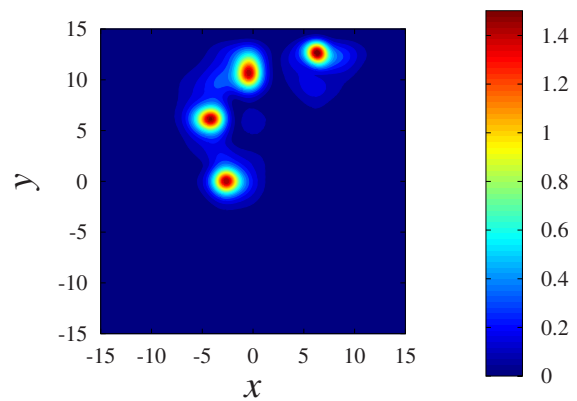

(f) $z=2642.9$

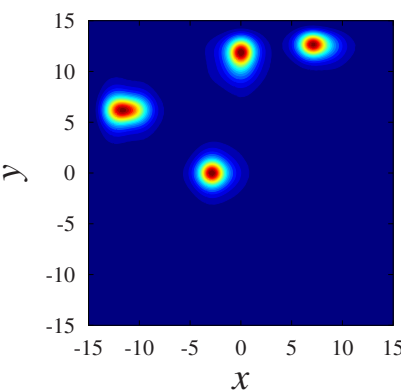

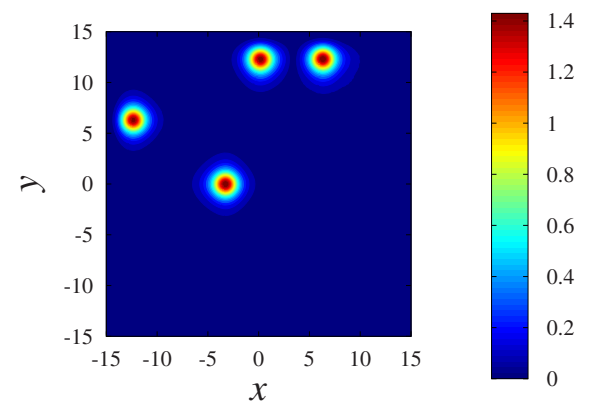

(h) $z=2708.7$

FIG. 14. (Color online) Evolution of an unstable vortex in the case of a small amplitude of the lattice potential, $V_{0}=0.15$. Other parameters are fixed as in Eq. (7).
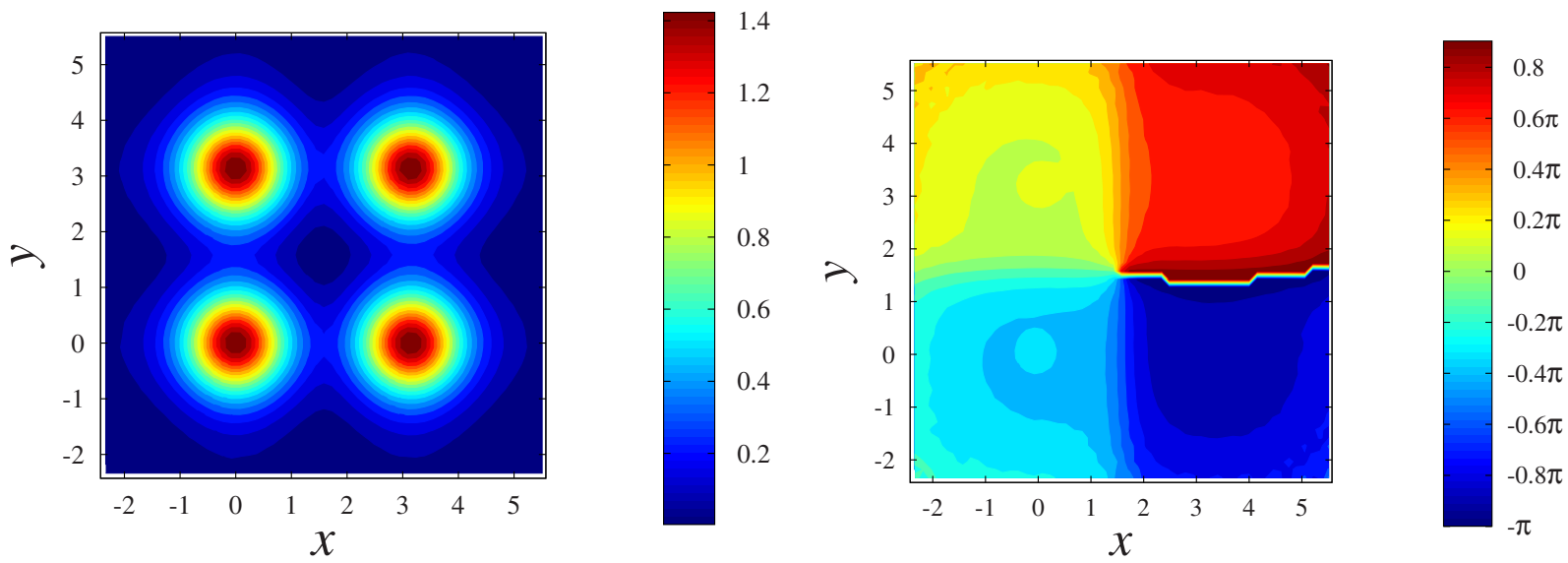

FIG. 15. (Color online) The amplitude and phase structure of a stable square-shaped (offsite) vortex found at values of parameters taken as in Eq. (7). 

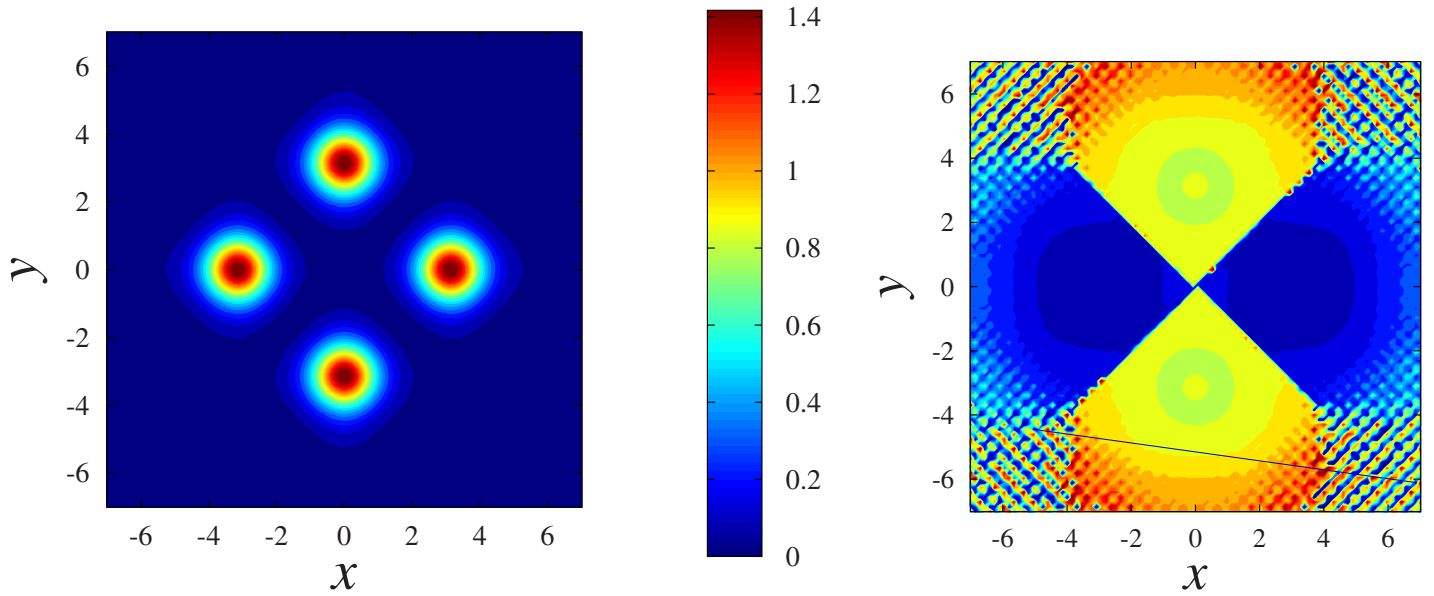

$0.8 \pi$

$0.6 \pi$

$0.4 \pi$

$0.2 \pi$

0

$-0.2 \pi$

$-0.4 \pi$

$-0.6 \pi$

FIG. 16. (Color online) The same as in Fig. 15 but for a stable quadrupole.
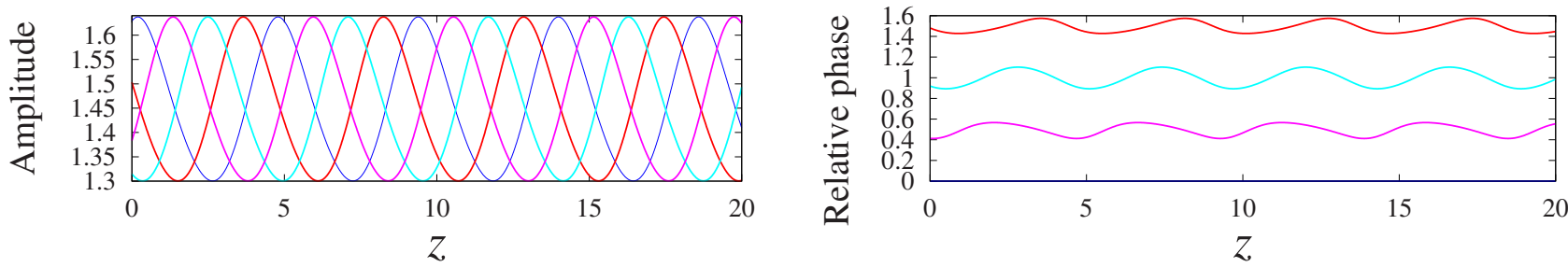

FIG. 17. (Color online) Regular oscillations of amplitudes of the four peaks which constitute a persistent vortical breather, and phase shifts between them, at $\nu=-0.53$ (just after the loss of the stability of the stationary vortex). Other parameters keep the same values as in Eq. (7).
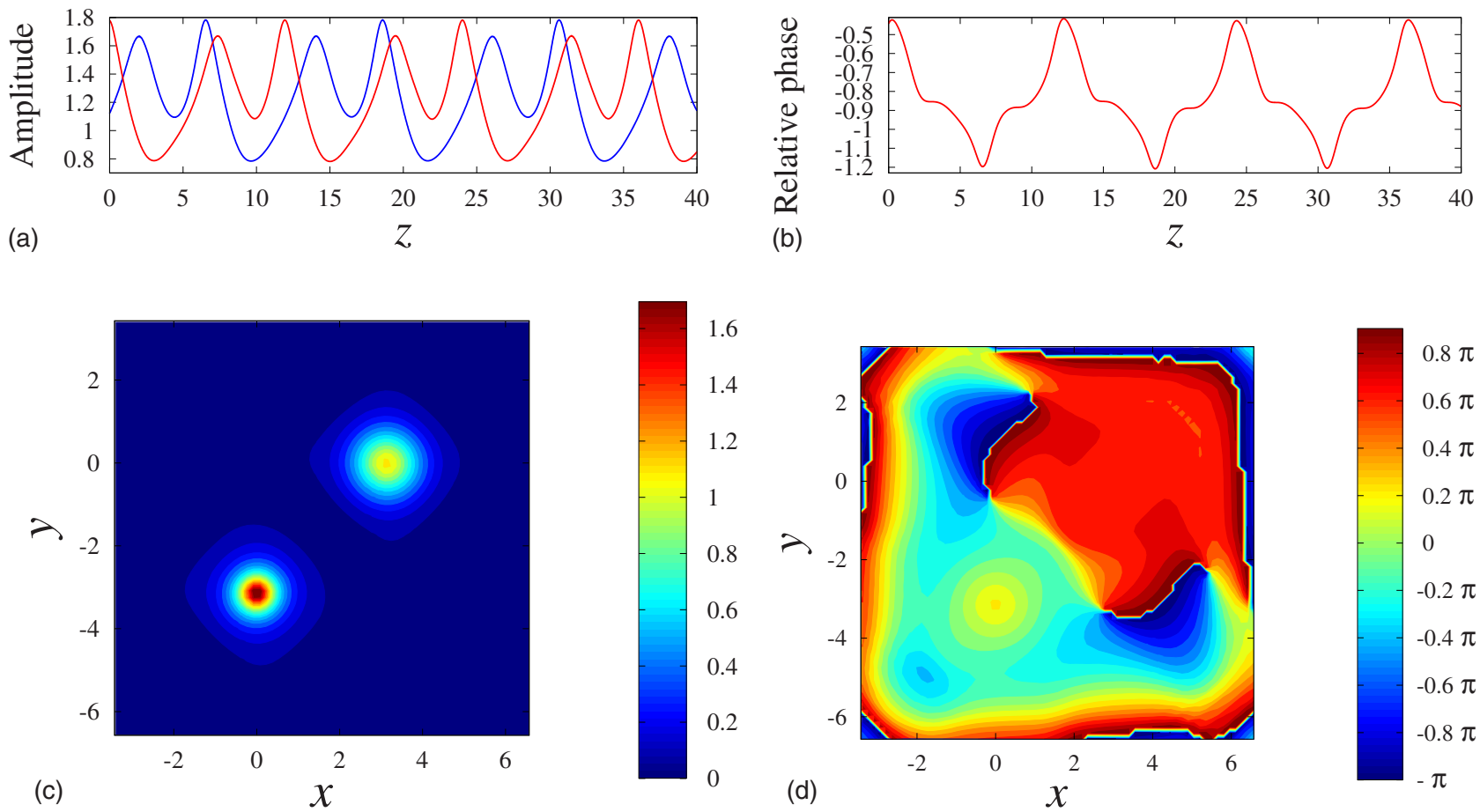

FIG. 18. (Color online) Right panels: a snapshot of the amplitude and phase distribution in a stable dipolar breather found at $\nu=-0.85$ (just after the vortical breather has lost its stability and rearranged into the dipole). Other parameters remain the same as in Eq. (7). Left panels display regular oscillations of amplitudes of the two peaks that constitute the breather and of the phase difference between them. 

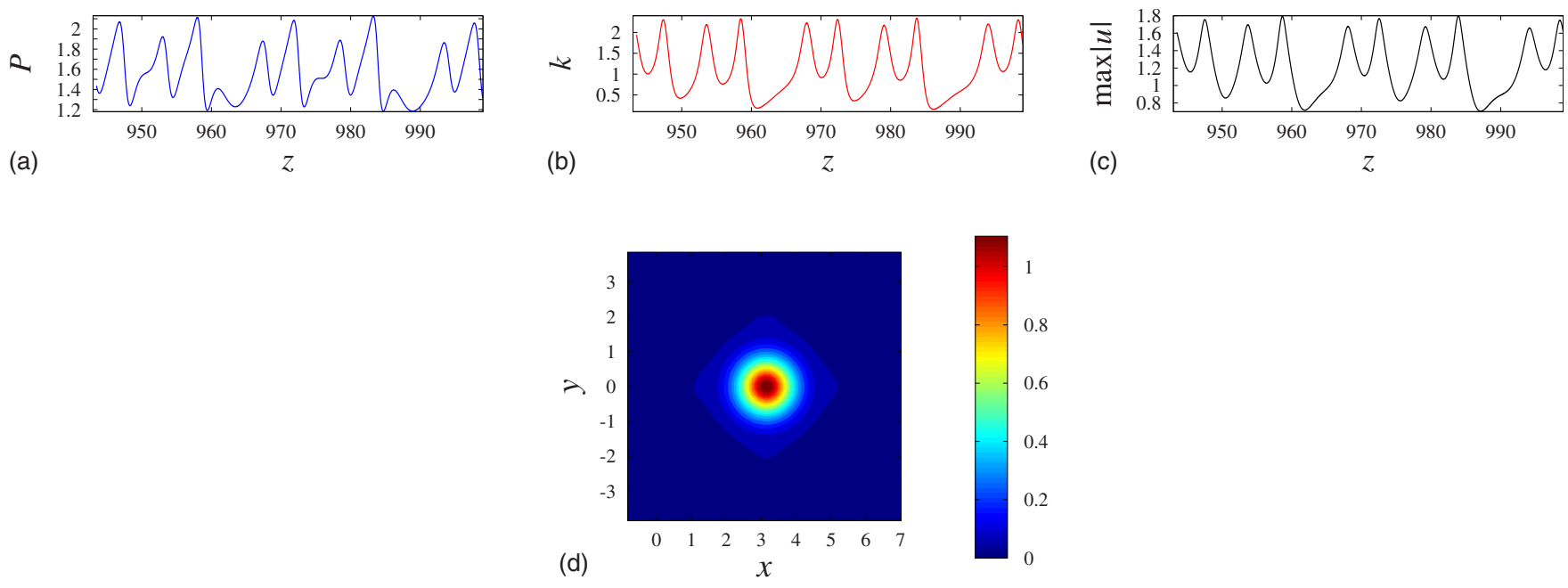

FIG. 19. (Color online) A stable single-peak breather found at $\nu=-0.882$ (after the dipolar breather has lost its stability). Other parameters remain the same as in Eq. (7).

ticity in the former case and the dipolar structure in the latter. At still larger values of $-\nu$, namely, $-\nu \geq 0.9$ [assuming, as before, that the other parameters are fixed as per Eq. (7)], any input decays to zero (a single peak may perform a few oscillations before disappearing).

\section{CONCLUSION}

This work aimed to introduce a 2D model of dissipative media, such as laser cavities, which would be able to support stable localized vortices under physically relevant conditions (i.e., without postulating an artificial diffusivity). We have demonstrated that this purpose may be achieved by means of a periodic lattice potential, which represents a transverse grating in the lasing medium. The analysis was chiefly reported for the vortex solitons of the onsite type, built as rhombuses, but offsite (square-shaped) vortices and rhombic quadrupoles were considered too. The stability region for the rhombic vortices, along with that for fundamental solitons, has been identified in the parameter space of the model, and generic scenarios of the evolution of the localized vortices have been explored in the case when the vortices are unstable. In particular, a minimum strength of the lattice potential necessary for the stabilization of the vortices has been found. If the potential is not strong enough, the vortex splits into a set of four uncorrelated fundamental solitons. On the other hand, if the instability is caused by "wrong" values of other parameters, the unstable vortex either decays to zero or spreads out into an irregular speckle pattern covering the entire domain.

The stability of the vortex solitons was also analyzed in the case when the conservative part of the quintic term in the CGL equation accounts for the self-focusing, which potentially may lead to the supercritical collapse in the 2D setting. A stability border for the vortices has been found in this case. If the strength of the quintic self-focusing exceeds the critical size, the stationary vortex is replaced by its oscillating counterpart (the vortical breather), which maintains the intrinsic vortical structure. Under still stronger self-focusing, the vortex breather is replaced by a dipolar breather and that one, in turn, is supplanted by a single-peak breather. Eventually, under very strong quintic self-focusing, all solutions decay to zero.

This work may be naturally extended in various directions. In particular, it may be interesting to study possibilities to stabilize higher-order vortices and "supervortices" (circular chains of compact vortices, with an independent global vorticity imprinted onto them), which should be based on more complex ring-shaped chains of localized peaks [31]. Another obviously interesting extension may be to consider the stabilization of localized vortical patterns in threedimensional CGL models.

\section{ACKNOWLEDGMENT}

B.A.M. and D.M. appreciate hospitality of ICFOInstitute of Photonic and Optical Sciences (Barcelona).
[1] N. N. Rosanov, Spatial Hysteresis and Optical Patterns (Springer, Berlin, 2002); P. Mandel and M. Tlidi, J. Opt. B: Quantum Semiclassical Opt. 6, R60 (2004); N. N. Rosanov, S. V. Fedorov, and A. N. Shatsev, Appl. Phys. B: Lasers Opt. 81, 937 (2005); C. O. Weiss and Ye. Larionova, Rep. Prog. Phys. 70, 255 (2007).
[2] Dissipative Solitons, Lecture Notes in Physics Vol. 661, edited by N. Akhmediev and A. Ankiewicz (Springer, Berlin, 2005).

[3] I. S. Aranson and L. Kramer, Rev. Mod. Phys. 74, 99 (2002); B. A. Malomed, in Encyclopedia of Nonlinear Science, edited by A. Scott (Routledge, New York, 2005), p. 157.

[4] B. A. Malomed, D. Mihalache, F. Wise, and L. Torner, J. Opt. 
B: Quantum Semiclassical Opt. 7, R53 (2005).

[5] A. S. Desyatnikov, Y. S. Kivshar, and L. Torner, Prog. Opt. 47, 291 (2005).

[6] V. I. Petviashvili and A. M. Sergeev, Dokl. Akad. Nauk SSSR 276, 1380 (1984) [Sov. Phys. Dokl. 29, 493 (1984)].

[7] B. A. Malomed, Physica D 29, 155 (1987); O. Thual and S. Fauve, J. Phys. (Paris) 49, 1829 (1988); S. Fauve and O. Thual, Phys. Rev. Lett. 64, 282 (1990); W. van Saarloos and P. C. Hohenberg, ibid. 64, 749 (1990); V. Hakim, P. Jakobsen, and Y. Pomeau, Europhys. Lett. 11, 19 (1990); B. A. Malomed and A. A. Nepomnyashchy, Phys. Rev. A 42, 6009 (1990); P. Marcq, H. Chaté, and R. Conte, Physica D 73, 305 (1994); N. Akhmediev and V. V. Afanasjev, Phys. Rev. Lett. 75, 2320 (1995); H. R. Brand and R. J. Deissler, ibid. 63, 2801 (1989); R. J. Deissler and H. R. Brand, ibid. 72, 478 (1994); 74, 4847 (1995); 81, 3856 (1998); V. V. Afanasjev, N. Akhmediev, and J. M. Soto-Crespo, Phys. Rev. E 53, 1931 (1996); J. M. SotoCrespo, N. Akhmediev, and A. Ankiewicz, Phys. Rev. Lett. 85, 2937 (2000); H. Leblond, A. Komarov, M. Salhi, A. Haboucha, and F. Sanchez, J. Opt. A, Pure Appl. Opt. 8, 319 (2006); W. H. Renninger, A. Chong, and F. W. Wise, Phys. Rev. A 77, 023814 (2008).

[8] L.-C. Crasovan, B. A. Malomed, and D. Mihalache, Phys. Rev. E 63, 016605 (2000); Phys. Lett. A 289, 59 (2001).

[9] P. Grelu, J. M. Soto-Crespo, and N. Akhmediev, Opt. Express 13, 9352 (2005).

[10] J. M. Soto-Crespo, P. Grelu, and N. Akhmediev, Opt. Express 14, 4013 (2006).

[11] V. Skarka and N. B. Aleksić, Phys. Rev. Lett. 96, 013903 (2006); N. B. Aleksić, V. Skarka, D. V. Timotijević, and D. Gauthier, Phys. Rev. A 75, 061802(R) (2007); V. Skarka, D. V. Timotijević, and N. B. Aleksić, J. Opt. A, Pure Appl. Opt. 10, 075102 (2008).

[12] J. M. Soto-Crespo, N. Akhmediev, and Ph. Grelu, Phys. Rev. E 74, 046612 (2006).

[13] D. Mihalache, D. Mazilu, F. Lederer, Y. V. Kartashov, L.-C. Crasovan, L. Torner, and B. A. Malomed, Phys. Rev. Lett. 97, 073904 (2006); D. Mihalache, D. Mazilu, F. Lederer, H. Leblond, and B. A. Malomed, Phys. Rev. A 76, 045803 (2007); 75, 033811 (2007).

[14] D. Mihalache, D. Mazilu, F. Lederer, H. Leblond, and B. A. Malomed, Phys. Rev. A 77, 033817 (2008); Phys. Rev. E 78, 056601 (2008); Eur. Phys. J. Spec. Top. 173, 245 (2009).

[15] D. Mihalache, D. Mazilu, L. C. Crasovan, I. Towers, A. V. Buryak, B. A. Malomed, L. Torner, J. P. Torres, and F. Lederer, Phys. Rev. Lett. 88, 073902 (2002).

[16] J. Lega, J. V. Moloney, and A. C. Newell, Phys. Rev. Lett. 73, 2978 (1994); Physica D 83, 478 (1995).

[17] A. Szameit, J. Burghoff, T. Pertsch, S. Nolte, and A. Tünner- mann, Opt. Express 14, 6055 (2006).

[18] J. W. Fleischer, M. Segev, N. K. Efremidis, and D. N. Christodoulides, Nature (London) 422, 147 (2003).

[19] H. Leblond, M. Salhi, A. Hideur, T. Chartier, M. Brunel, and F. Sanchez, Phys. Rev. A 65, 063811 (2002); A. Komarov, H. Leblond, and F. Sanchez, Phys. Rev. E 72, 025604(R) (2005); M. Salhi, A. Haboucha, H. Leblond, and F. Sanchez, Phys. Rev. A 77, 033828 (2008).

[20] B. B. Baizakov, B. A. Malomed, and M. Salerno, Europhys. Lett. 63, 642 (2003).

[21] T. Mayteevarunyoo, B. A. Malomed, B. B. Baizakov, and M. Salerno, Physica D 238, 1439 (2009).

[22] J. Yang and Z. H. Musslimani, Opt. Lett. 28, 2094 (2003); J. Wang and J. Yang, Phys. Rev. A 77, 033834 (2008).

[23] A. Ferrando, M. Zacarés, P. Fernández de Córdoba, D. Binosi, and J. A. Monsoriu, Opt. Express 12, 817 (2004).

[24] M. I. Rodas-Verde, H. Michinel, and Y. S. Kivshar, Opt. Lett. 31, 607 (2006).

[25] D. N. Neshev, T. J. Alexander, E. A. Ostrovskaya, Y. S. Kivshar, H. Martin, I. Makasyuk, and Z. Chen, Phys. Rev. Lett. 92, 123903 (2004); J. W. Fleischer, G. Bartal, O. Cohen, O. Manela, M. Segev, J. Hudock, and D. N. Christodoulides, ibid. 92, 123904 (2004).

[26] A. I. Yakimenko, Y. A. Zaliznyak, and Y. Kivshar, Phys. Rev. E 71, 065603(R) (2005); D. Briedis, D. E. Petersen, D. Edmundson, W. Królikowski, and O. Bang, Opt. Express 13, 435 (2005); A. I. Yakimenko, V. M. Lashkin, and O. O. Prikhodko, Phys. Rev. E 73, 066605 (2006); S. Skupin, M. Saffman, and W. Królikowski, Phys. Rev. Lett. 98, 263902 (2007); Y. V. Kartashov, V. A. Vysloukh, and L. Torner, Opt. Express 15, 9378 (2007); Phys. Rev. A 79, 013803 (2009); A. A. Minzoni, N. F. Smyth, A. L. Worthy, and Y. S. Kivshar, ibid. 76, 063803 (2007); Y. J. He, B. A. Malomed, D. Mihalache, and H. Z. Wang, ibid. 77, 043826 (2008); W. P. Zhong and M. Belić, ibid. 79, 023804 (2009); R. M. Caplan, Q. E. Hoq, R. Carretero-González, and P. G. Kevrekidis, Opt. Commun. 282, 1399 (2009).

[27] C. Rotschild, O. Cohen, O. Manela, M. Segev, and T. Carmon, Phys. Rev. Lett. 95, 213904 (2005).

[28] L. Bergé, Phys. Rep. 303, 259 (1998).

[29] W. J. Firth and D. V. Skryabin, Phys. Rev. Lett. 79, 2450 (1997); L. Torner and D. V. Petrov, Electron. Lett. 33, 608 (1997); D. V. Petrov, L. Torner, J. Martorell, R. Vilaseca, J. P. Torres, and C. Cojocaru, Opt. Lett. 23, 1787 (1998).

[30] J. Gómez-Gardeñes, B. A. Malomed, L. M. Floría, and A. R. Bishop, Phys. Rev. E 74, 036607 (2006).

[31] H. Sakaguchi and B. A. Malomed, Europhys. Lett. 72, 698 (2005). 